\title{
Entretien avec Georges Lavaudant : mise en scène de Richard III
}

Georges Lavaudant

\section{CpenEdition}

Journals

Édition électronique

URL : http://journals.openedition.org/shakespeare/528

DOI : $10.4000 /$ shakespeare. 528

ISSN : 2271-6424

Éditeur

Société Française Shakespeare

Édition imprimée

Date de publication : 1 novembre 1989

Pagination : 163-170

Référence électronique

Georges Lavaudant, "Entretien avec Georges Lavaudant : mise en scène de Richard III 》, Actes des congrès de la Société française Shakespeare [En ligne], 6| 1989, mis en ligne le 01 janvier 2007, consulté le 24 avril 2019. URL : http://journals.openedition.org/shakespeare/528 ; DOI : 10.4000/ shakespeare.528 


\section{SOCIETE FRANCAISE SHAKESPEARE}

\section{Actes du Congrès - 1984}

\section{LIEU ET TEMPS}

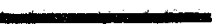

Directeur de la publication Jean FUZIER 


\section{Entretien avec Georges LAVAUDANT "Mettre en scène Richard III "}

Q. Pourquoi ce choix de Richard III puisqu'il y avait eu un premier travail ? Peut-être doit-il y avoir une certaine fascination pour la pièce, le personnage?

G.L. C'est une histoire qui démarre en 1974. Lorsqu'on est une jeune compagnie sans trop de moyens, avec quatre ou cinq comédiens on se réunit pour savoir quelle pièce on va monter, on tourne autour de Marivaux, et tout d'un coup, Ariel GarciaValdes, qui jouera Richard III, renverse la problématique : plutôt que de choisir une pièce qui soit à la hauteur de nos moyens, faisons la chose que l'on ne devrait jamais faire: montons la pièce qu'il est impossible de monter quand on n'a ni les moyens, ni l'argent, ni les acteurs, ni la force de travail nécessaires. Et donc en 1974, au lieu de monter un petit spectacle avec quatre acteurs, on décide de monter Le Roi Lear dans des conditions très compliquées. Cela a été notre premier coup de foudre pour Shakespeare qu'on ne connaissait pas du tout. A l'époque, on ne se posait pas de problèmes, de traduction par exemple. On a disposé du matériau à notre gré puisqu'on l'a abordé sans glose, sans barrière, on a touché à tout ce qui nous 
intéressait très profondément. On n'a pas de tradition littéraire ou universitaire. Je ne nie pas l'étude et dès que je peux m'y plonger, cela me passionne, mais dans l'ensemble on est une troupe plutôt sauvage, et on a toujours eu un point aveugle: celui de refuser le répertoire classique français. Il y a bien sûr l'exception qui confirme la règle : en 1975 on a monté Lorenzaccio. Depuis, on n'y est jamais revenu. On a toujours évité ce gros corpus de la langue française préconstitué du XVIIe, XVIII ${ }^{\mathrm{e}}$ et même du XIX ${ }^{\mathrm{e}}$. Les autres textes, (à part les contemporains qu'on a montés), Pirandello, Brecht, Shakespeare, avaient toujours ce problème de traduction qui nous les faisait entendre presque comme des textes contemporains. Je ne suis pas certain d'entendre la langue de Shakespeare comme on devrait l'entendre, comme du Rabelais, du Montaigne... Dans ce cas, je ne suis pas certain que je monterais du Shakespeare. Mais comme la traduction, malheureusement peut-être, mais heureusement pour moi, me fait entendre la pièce presque comme un texte contemporain, c'est donc quelqu'un qui nous passionne totalement. C'est un rapport que l'on voudrait continuer avec Shakespeare, bien que ce soit un rapport un peu Bâtard, et qui le restera, il me semble, puisque cela me convient.

En 1979, un certain nombre de comédiens travaillaient avec André Engel sur Penthésilée et pour d'autres, comme Ariel Garcia-Valdes, j'ai proposé de travailler sur l'adaptation de Carmello Bene de Richard III (aux Editions de Minuit, Superpositions, suivi d'un texte de Deleuze sur la manière dont Carmello Bene conçoit le théâtre). Donc à partir de cette petite version, avec des costumes et des décors qu'on avait récupérés de Hamlet, on afait ce premier spectacle qui s'appelait La Rose et la Hache, qui était un peu tiré d'une pensée de Cioran. Cette version est très réduite puisqu'elle ne concerne que Richard III et les femmes - Lady Ann, Margaret, Elisabeth -, et Buckingham, joué par une femme dans la version de Carmello Bene, c'est une espèce de servante. On a fait ce travail à quatre, et déjà Ariel Garcia-Valdes jouait Richard III. C'était un travail très heureux car il était très irrévérencieux. Comme il était à perte, on a vraiment fait les pieds au mur, on s'amusait avec le texte. 
En 1984, après avoir fait Les Céphéides, à Avignon, Bernard Faivre d'Arcier, le directeur du festival, a insisté pour qu'on rejoue en Cour d'Honneur. On avait un certain nombre de projets qui étaient déjà mis en route, on s'était dit que ce serait très difficile de faire une création complète à Avignon et qu'il vallait mieux s'appuyer sur un travail qu'on avait déjà ébauché. D'où le choix très forain de Richard III - c'est à dire un choix très ponctuel : avoir l'acteur sous la main - Ariel Garcia-Valdes -, et l'argent pour monter le spectacle. Bien que tenté, je ne me sentais pas la force de présenter les comédies de Shakespeare ou du Tchékov dans la Cour d'Honneur qui est un endroit très particulier au point de vue architectural. L'autre choix, ce sont les dix pièces que tout metteur en scène a dans sa tête et qu'il tourne sans cesse et dont l'ordre de tirage est le hasard des circonstances concrètes. Pour moi, il y a L'Illusion Comique, une ou deux comédies de Shakespeare, Oncle Vania, La Tempête, une ou deux pièces de Brecht: Baal ou Tambours dans la Nuit... Je sais que de toutes façons je les monterais, mais l'ordre est plus directement amené par des circonstances très foraines. Je ne nie pas du tout le côté terre à terre que peut avoir parfois le théâtre. Ce serait mentir que de dire qu'un metteur en scène monte une pièce parceque c'est uniquement celle-là qu'il voulait monter. Un metteur en scène a toujours deux ou trois projets devant lui, et il en choisi un, à un moment donné, comme un coup de poker. Richard III s'est un peu fait comme cela.

Et la musique? Cela a été une question polémique. Pourquoi les mandolines, par exemple? Elles viennent directement de Carmello Bene dans les notes de son introduction : Richard III il faut le jouer comme sur une plazza italienne, on mange des fruits de mer et on entend des mandolines. Je trouvais que c'était une bonne idée : la cour s'ennuie - un air revient sans arrêt. On a aussi voulu remettre de façon mécanique certaines musiques que l'on avait choisies pour La Rose et la Hache, et qui ne sont pas forcément les choses qui fonctionnent le mieux, ou certaines même, ne fonctionnent pas. Mais cela a été très dur de s'arracher à ce souvenir.

Q. L'interprétation de Richard a-t-elle été influencée par ce spectacle antérieur? 
G.L. Non. Dès qu'on a commencé les répétitions de Richard III, Ariel avait déjà le rôle. Son problème était le suivant : comment répartir son effort qu'il avait dosé sur une heure et quart, dans la version de La Rose et la Hache, sur trois heures et demi. Il y avait d'autres problèmes, de tempo par exemple - savoir s'effacer, en particulier dans la deuxième moitié de la première partie : pendant une heure, il n'y a pratiquement que Richard en scène, c'est lui qui mène la danse. Il y a un moment tactique qui est formidable: Richard a vraiment un peu trop occupé le devant de la scène, à un moment, il arrive à s'effacer et à mettre en avant Buckingham. Il y a une espèce de jeu de passe-passe où Richard sent très bien le moment où il sature un peu. Et dans cette deuxième moitié, tout d'un coup, c'est plutôt Buckingham qui devient le personnage politique du spectacle, quasiment jusqu'à l'entr'acte. Pendant ce temps, Richard joue un peu les mijaurées, disant "je suis là ", " je ne suis pas là ». Mais c'est Buckingham qui mène l'affaire. C'est plus cette partie qui est compliquée à traiter car, à tous les niveaux, tant de l'acteur, des spectateurs que du metteur en scène, dès que Richard n'est plus là, on s'ennuie (moi, en tous cas, je m'ennuie). Cette complication est à double plan, celle du metteur en scène et de l'acteur, pour doser son effort. Je le redis encore une fois, dans La Rose et la Hache, Richard était en scène du début jusqu'à la fin, il ne quittait pas la table (c'était le dispositif scénique), c'était plus le rêve de la dernière journée, à la veille de la bataille, quand on trouve Richard en attente, à la fin, dans le dernier acte, où il demande sans cesse: "donnez-moi une coupe de vin", "que vais-je faire, les ombres arrivent ". C'était plus cette dernière journée avant le combat. Alors que là, il faut quand même reprendre la pièce dans la durée. Il y avait donc un problème de tempo à travailler avec Ariel. Là aussi, je pense que certains passages sont bien, et d'autres moins bien.

Q. Dans les deux phases du projet, il y a un choix significatif, répété : prendre, pour interpréter ce personnage de boîteux, d'estropié, d'homme laid, un acteur beau, un acteur plutôt fait pour jouer les séducteurs que les nabots bossus et difformes.

G.L. Oui, mais on a eu la chance de voir les deux choses avec Jean-Michel Deprats. A Lille s'est monté il y a à peu près six 
mois un Richard III ré-écrit par Chartreux qui s'appelait Cacodémon Roi où le parti-pris était absolument l'inverse. Très nettement, Alain Milianti qui était le metteur en scène et qui est souvent l'assistant de Gildas Bourdet, a pris un acteur laid physiquement. On sentait qu'il voulait affirmer un corps laid. Mais comme c'est donné d'emblée, vous avez un quart d'heure qui est absolument faramineux parce que tout d'un coup, ça vous impose une autre image que celle que vous attendez, et au bout d'un moment, on retombe tout de même sur le problème de l'acteur: est-il bon ou pas bon? Il ne s'agit pas simplement de le choisir beau ou laid, il s'agit de savoir si l'acteur est capable de travailler.

Q. Cela a son importance. Mais le rapport que peut avoir le spectateur pendant au moins une heure est un rapport de répulsion très marquée, alors que dans votre Richard III, il y a immédiatement un rapport de fascination, d'identification. Cela change quand même le rapport au monstrueux.

G.L. Oui, mais c'est peut-être toujours la même idée. Depuis trois ou quatre ans, je pense que quand on met sur scène des personnages quels qu'ils soient, on doit leur donner leur chance. Je me suis fait une idée que tout personnage qui apparaît sur une scène de théâtre doit avoir sa chance et même plus.

Q. Vous avez raison de dire que quand Richard n'est pas là, on s'ennuie. Ce que j'ai le moins compris, c'est le virage sur la fin, cette espèce d'image d'Epinal avec ces chevaux mécaniques. A la fin seulement, pas quand Richard accède au pouvoir, parce que là, il reste lucide et presque dérisoire par rapport au propre roi. Je reparlerai un peu de Richard II de Mnouchkine. Ce qui m'avait un peu gêné dans cette mise en scène, indépendamment des masques qui étaient très beaux, c'était le kabouki. Ce que je reconnais dans votre Richard III, c'est que tout passe, ce qu'il dit et ce pour quoi il le dit. Mais à la fin, avec les chevaux mécaniques et le H.F., on a l'impression que le langage n'appartient plus au personnage.

G.L. Je n'ai là que des réponses qui n'en sont pas vraiment. Dans la version de salle on a réduit l'utilisation du H.F. Elle ne reste qu'à la fin, mais elle n'est plus sur la durée du cinquième acte. 
Dès le départ, je le savais, je voulais des chevaux. J'ai horreur du naturel sur la scène, je me le suis toujours interdit, je n'ai jamais mis un animal sur scène. Je reconstituerai toujours le naturel. Et c'est vrai que le cinquième acte a été composé d'une manière un peu figée, artificielle. On a une espèce d'alphabet de mise en scène qui est pris ailleurs.

J.M. Deprats Dans la pièce elle même, il y a intervention d'artifice dans l'écriture: des conventions du théâtre médiéval, avec une tente de chaque côté, une alternance entre Richmond et Richard. Il y a un changement de ton, d'écriture.

G.L. Très objectivement, c'est juste ce que vous dites. On fait certaines choses en se disant que c'est peut-être faux, mais on les fait quand même. Je dois dire que c'était presque la partie que j'amais le mieux.

J.M.D. Ce n'est pas du tout pour légitimer la mise en scène, mais il y a des espèces d'images d'Epinal dans la façon dont est représenté Richmond.

G.L. Oui, en tous cas, le cinquième acte est très ritualisé.

Q. Je voudrais revenir sur la séduction de Richard III. J'ai l'impression que le spectacle était axé sur la solitude de Richard III. Ce qui fait qu'on ne donnait pas tellement à voir les modes et les modalités de la séduction. Je voulais savoir si c'était délibéré. En particulier les femmes ne donnaient pas à voir aux spectateurs ce qui se passait en elles au contact du monstre. Richard III restait dans une sorte de solipsisme, privé de tout échange avec l'entourage.

G.L. Dans ce que vous dites, il y a un double problème : il y a un problème d'acteurs et d'actrices. Il faut que les acteurs soient aussi à la hauteur du travail qu'on leur demande. Et il y a ensuite ce qui accentue la mise en scène dans cette chose-là. Il est vrai que je crois qu'il n'y a que Richard dans cette pièce, tous les autres sont un peu comme des papillons de nuit qui viennent se brûler autour de lui. Elisabeth est la seule qui, à la limite, soit un personnage qui a un parcours. Les autres viennent et puis repartent après s'être brûlés. Je ne sais pas s'il y a vraiment une possibilité de faire qu'un personnage ait un échange 
avec Richard. Personnellement, l'échange, je le voyais plus avec Buckingham. C'est une option personnelle.

Pierre Sahel Je voudrais reprendre la question des personnages, au moment où ils s'affrontent, durant la bataille. Il y a quelque chose de très conventionnel dans ces grands chevaux, qui vont à la rencontre l'un de l'autre, qui donnent quelque chose d'assez figé. Il y a aussi une utilisation des micros à la fin de la pièce qui ne signifie plus grand chose. Juste avant la charge des deux chevaux, qu'avez vous voulu dire avec l'image athlétique d'un Richard et d'un Richmond accompagnés de leurs soigneurs?

G.L. Quand on fait du théâtre, il y a des tics qui reviennent, des choses que vous faites quasiment toujours dans tous les spectacles. Si je les avais faites jusqu'au bout, j'aurais fait ces personnages presque comme des matadors. Ce qui m'intéressait, c'était cette espèce de peu et de solitude, quand vous devez jouer votre destin sur un coup, malgré les gens qui sont autour de vous. C'est comme un boxer avant d'entrer, il a beau avoir dix managers autour de lui, les frères, la famille qui lui disent "tu es bon ", " tu es le meilleur ", " on a prié ", il y a quand même l'idée que quelqu'un est là, seul, et a rendez-vous avec son destin. C'est moins le côté athétique, musclé, que ces gens-là qui, de toute façon, sur une épreuve physique, doivent livrer un combat qui aura des répercussions morales. On les masse, on les enrobe, on leur donne à boire.

P.S. Mais vous n'avez pas été jusqu'au bout. Je trouve que cette image de boxers est très bien et très originale. Et puis ensuite, ils sont là, avec des canassons. Pour moi, il y a une chute, et même pire, il y a juxtaposition de deux images. Il me semble que vous n'avez pas poussé jusqu'au bout une idée qui vous était chère, je le regrette.

Q. Le fait que toute la pièce se joue sur une scène si large est déjà un parti-pris parce que les acteurs sont complètement perdus, là, au milieu. On peut considérer les acteurs comme des marionnettes perdues.

G.L. Il y a deux solutions pour Avignon : soit reconstituer dans la cour un dispositif scénique qui sera toujours en trop, remettre 
un petit décor au milieu de cet espace extraordinaire, c'est à cela que l'on se heurtait. J'avais posé à J.P. Vergier la question inverse : non pas que l'on allait jouer à Avignon - on sait que c'est blocant, on ne trouve nulle part en France un pareil espace -, à l'inverse, je lui avait demandé de partir du dispositif de salle, et ensuite comment ce dispositif pourrait se redéployer à Avignon. Cela a été impossible. Chaque fois, on avait l'impression de remettre un petit truc minuscule dans l'espace d'Avignon. Et j'ai préféré jouer l'inverse. : il y a cet espace, eh bien, on est capable de le prendre. Alors après, c'est jugé différemment : certains disent qu'on s'y perd. Personnellement, je ne trouve pas. Je trouvais qu'il y avait une certaine respiration des choses que je trouvais belle. Bien sûr, il y a plein de choses critiquables dans la mise en scène, mais je trouvais que justement, ce problème était résolu. Cela ne vient pas du dispositif scénique mais des salles, quand il y a deux mille ou deux mille cinq cents places. A mon sens, une salle ne devait pas dépasser six cents places. Au-delà, quand on est placé derrière, on ne voit plus l'acteur mais sa silhouette, et là c'est fini. A Avignon, c'est encore pire: si vous faites une mise en scène pour les premiers rangs, derrière on ne voit plus rien, et à l'inverse, si vous la faites pour la deuxième moitié, les premiers spectateurs peuvent se demander pourquoi les acteurs jouent ainsi.

Q. Vous avez choisi un style de jeu qui est tout à fait ritualisé et qui est déjà une stylistique.

G.L. On va reparler d'Ariane Mnouchkine. Je ne suis pas entièrement d'accord avec ses options, mais au moins, elle a résolu le problème stylistiquement - ce qui est fait est clair. Ce n'est pas gênant ni devant ni au fond, c'est assumé comme tel. Si vous hésitez, et on continue d'hésiter au niveau du jeu psychologique, si vous voulez jouer très honnêtement, réel, sincère, si l'acteur ne ritualise pas mais joue intériorisé, le spectateur au deuxième gradin d'Avignon se demande ce qui se passe, il voit une tache blanche, ce n'est pas l'idéal. A Avignon, on n'arrive pas à résoudre ce problème si on n'affirme pas un volontarisme stylistique. C'est ce que je ressens. J'ai vu le spectacle des deux endroits... et au deuxième gradin... je dormais et j'attendais que ça soit terminé. On ne voit rien, on n'entend rien, si l'acteur hurle, les gens qui sont devant se demandent pourquoi ! 